\title{
Research on distributed price monitoring system based on Multi-Agent
}

\author{
Bi Puyun \\ Department of Economics Research, Hainan Province School of \\ Administration, \\ Haikou, 571100, China \\ email:1046519082@qq.com
}

\begin{abstract}
The paper puts forward new ideas for the establishment of distributed price monitoring system on based on the Multi-Agent theory, in order to make the price monitoring process intelligent, the fault diagnosis of real-time price intelligent and conflict resolution intelligent. Price monitoring intelligent system based on Multi-Agent, which realize the intelligent operation, control and supervision of prices, can improve the decision of the whole control system, and improve the quality of the price data. The intelligent system not only provides the technical support for the price monitoring in China, but also explores a feasible technique approach for the Multi-Agent theory.

Keywords: Multi-Agent; price monitoring system; Bottom platform
\end{abstract}

\section{Introduction}

With the Chinese government investment scale increasing and super currency behavior to reverse the economic decline in some countries, Inflation occurs in most emerging economies especially China. Due to the present price monitoring system has many problems, the price index and the people's real feeling still has the big disparity. The people desire the real increasing extent of price, and more effective ways to monitor the price. How to use artificial intelligence technology to achieve price monitoring process stability, and ensure the monitoring data and results reliability, has become urgent.

Bottom platform

intelligent distributed price monitoring system based on Multi-Agent

In recent years, many foreign famous companies have entered the Chinese price monitoring automation. The price monitoring of China's automatic control system has reached the average level of the world. It is the main trend for the structure of the control system become intelligent, networked and open. However, establish intelligent distributed price monitoring system of Multi-Agent depend 
on the construction of a real-time, distributed computing environment. The Agent in network needs the autonomy, and distributed network environment can provide an interactive mechanism for the coordination, cooperation between each Agent. At present, the distributed architecture based on DCS provides a fully distributed control computing environment. Field controller in the control system (PLC) which is equivalent to an Agent has the autonomy, which can supply the interoperable interaction mechanism for the scenes Agent. DCS system structure combined natural division and hierarchical of divided artificially. We think, the intelligent system based on Multi-Agent has a distributed hierarchical structure can play an important role in price monitoring.

\section{Architecture of distributed price monitoring intelligent system based on Multi-Agent}

The locale control layer is composed of distributed consists of PLC in production site, which can realize the field parameter collection and automatic control. It corresponds to the Agent of execution. the data layer provides access function to real time database in process control system, corresponding to the intelligent data service in Agent system. The knowledge layer realizes function of information inference and decision, which includes conflict resolution Agent and fault diagnosis Agent. The management layer can increase, filtrate and update information, which corresponds to the agent of knowledge management. Agent of communication management layer is responsible for maintaining the whole system platform, and the Agent it is the price monitoring infrastructure in multi Agent system.

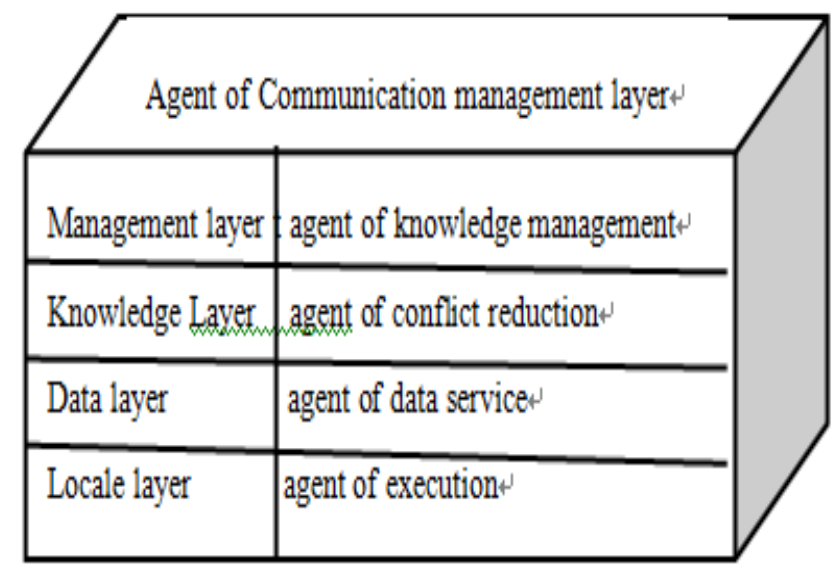

Fig.1. The hierarchical structure of the system

The model structure of intelligent price monitoring system based on Multi-Agent as shown in Figure 1. Each Agent completes a specific task cooperatively in the system environment according to their respective work. 


\section{The model structure of price monitoring system based on Multi-Agent}

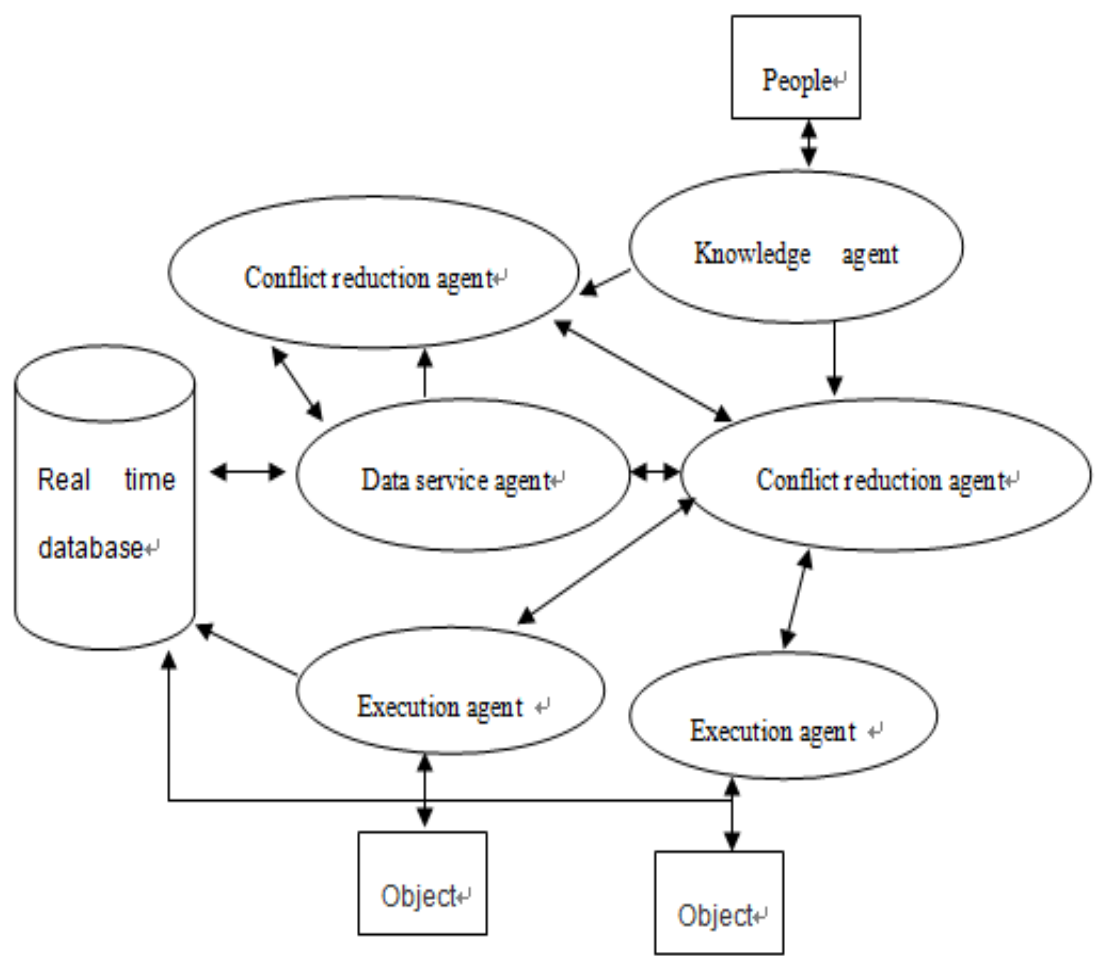

Fig.2. The distributed model structure of price monitoring system based on Multi-Agent

The various price monitoring parameter value and the set value of the controlled variable stored in real time database (established by the automatic control system). We can write parameter values to guide the operation of the system, also get real-time data to achieve the purpose of control. Data services agent dedicated to provide the real-time database, which the only agent is dealing with data in the whole system. Other agents finish the read and write operations by data service agent. In price monitoring process, because of the environmental disturbance uncertainly and correlation between the controlled parameters, control of certain parameters in the system may conflict directly or indirectly with other control action. Conflict resolution in Agent system will be monitored for possible conflict phenomenon. When conflict happens, it sends out instructions to perform conflict resolution related to Agent, such as the suspension of execution actions, adjustment of the setting value. When the system has abnormal data, fault diagnosis of Agent will carry on logic reasoning according to the domain expert knowledge, to find out the possible causes, and give guidance. At the same time, it also accepts fault diagnosis service from other agents any time. Knowledge Agent is responsible for the expert knowledge. 
Execution agent is executed by the corresponding implementation of agent.

\section{Scheme of integrated price monitoring intelligent system and automatic control system}

RS View, a software product from equipment provider Rockwell Automation Company, provides the interface for automatic control system and other system integration using OPC technology. In the integration of MAS and automatic control system, RS View is an OPC server, synchronous monitoring program is an OPC client, and synchronous monitoring program can read and write any value through the command transmission between them. On the other hand, synchronous monitoring program operates data in MAS, and ultimately realizes two system data synchronization. In this way, the proposed MAS price monitoring intelligent system can be easily integrated with any automatic control system.

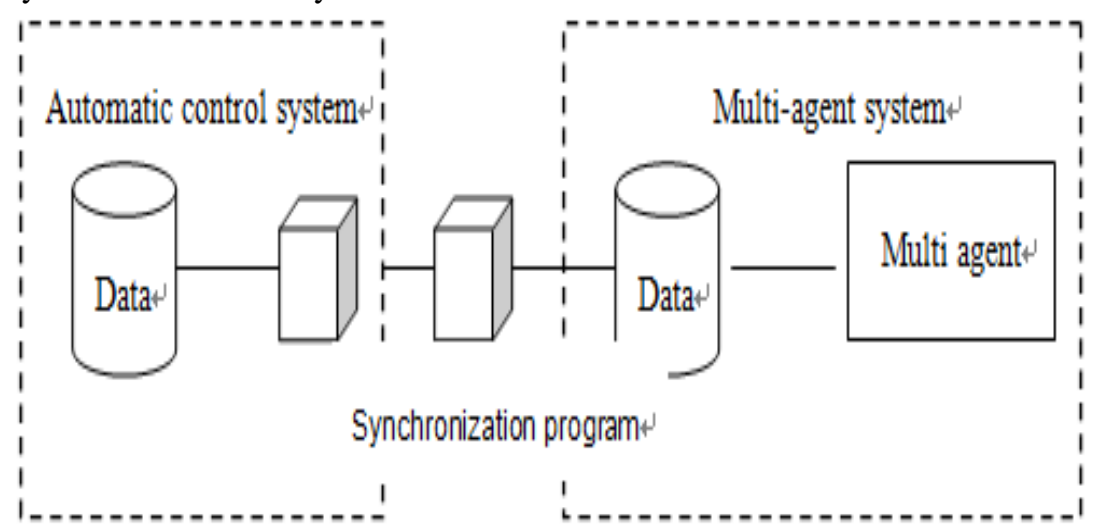

Fig.3. The integration structure of intellectualized system and supervise system

\section{Conclusion}

Price monitoring is a complicated process. It is difficult to realize the stable operation by the traditional control theories and technologies. At present, research on the application of artificial intelligence technology in engineering system, has become the hot application fields, but its application in the field of price monitoring in China is still at the exploratory research stage. In this paper a more comprehensive analysis proposed the establishment of the new idea of Multi-Agent theory of price monitoring based on distributed intelligent system, to achieve the process of intelligent control.

\section{Acknowledgement}

In this paper, the research was sponsored by the Nature Science Foundation 
of Hainan Province (Project No. 714279).

\section{References}

[1]Lucia Filgueiras, Plinio Aquino, Vera Tokairim, Carlos Torres and Iara Barbarian. Building the E-Service Society, International Federation for Information Processing[A], 2004.

[2]Jianjun Cheng, Sencheng Cheng and Meiju Yang,Evaluating the E-government Based on BSC, Integration and Innovation Orient to E-Society [A], 2007..

[3]Olsson G, Aspegren H,Operation and control of wastewater treatment -a Scandinavian perspective over 20 years. Water Science and Technology [J], 1998.

[4]Karl Tuyls, Ann Nowe, Zahia Guessoum.Adaptive Agents and Multi-Agent Systems III. Adaptation and Multi-Agent Learning 5th, 6th, and 7th European Symposium [A], 2007.

[5]Tom Wagner, Omer F. Rana, Infrastructure for Agents, Multi-Agent Systems, and Scalable Multi-Agent Systems, International Workshop on Infrastructure for Scalable Multi-Agent Systems[J], 2009.

[6]Magnus Boman, Walter Van de Velde, Multi-Agent Rationality, 8th European Workshop on Modelling Autonomous Agents in a Multi-Agent World [A] , 1997.

[7] Maja Hadzic, Pornpit Wongthongtham, Tharam Dillon,Elizabeth Chang, Ontology-Based Multi-Agent Systems, Studies in Computational Intelligence[J], 2009 